\title{
Microsecond time scale thermocouple temperature measurements in solid penetrated by a shaped charge jet
}

\author{
Juxian Gao and Rongshang Bai \\ Institute of Mechanics, Academia Sinica, P. O. Box 2251, Beijing 100080, People's Republic of China
}

(Received 16 March 1992; accepted for publication 8 November 1994)

\begin{abstract}
The development of mictosecond time scale temperature measurements in shock-loaded solids is briefly reviewed. The feasibility of making thermocouple temperature measurements near a cavity created by a shaped charge jet in medium carbon steel and glass fiber reinforced epoxy composite solid targets has been studied. Thermal diffusion calculations demonstrated that these measurements were feasible if the foil thermocouple was on the order of $600 \AA$ thick. Chromel-alumel thermocouples were used because of the anticipated temperature measurement range and also because they have a small pressure correction. The thermocouple fabrication method is discussed in this article. By embedding the thermocouples in the target plates at different distances from the shaped charge generated cavity, temperature distributions were obtained in the steel and composite materials. These temperature distributions were shown to be consistent with temperatures for melting at the cavity in the steel and for epoxy degradation in the composite material. (c) 1995 American Institute of Physics.
\end{abstract}

\section{INTRODUCTION}

In recent years there has been a recognized need to measure temperature directly in shock-compressed solids in order to determine experimentally a complete equation of state $^{1}$ to study chemical activity under shock-loading conditions, ${ }^{2}$ in our case, to understand the penetration dynamics of shaped charge jet. However, this is very difficult in solids. Some success in inferring the temperature of shockcompressed solids based on infrared radiation measurements has been reported. ${ }^{3}$ This method has been limited to cases where transparent materials were shocked to high temperatures; successful methods for measurements in opaque solids have not yet been found. ${ }^{4,5}$ Using thermally sensitive films ${ }^{6}$ to measure temperature was found to be only a qualitative method. A special thermocouple has been designed in order to reduce thermal inertia of the sensor, ${ }^{7}$ and has been used to measure temperature in explosive welding. ${ }^{8}$ This method has not been widely applied, because several problems still exist. For example, a space around the junction exists which will affect both the stress-strain and temperature fields. ${ }^{7}$

The measurement of temperature in shock-compressed solids using various foil materials and configurations has been studied in recent years. For example, 5 - $\mu$ m-thick foil thermocouples, ${ }^{9-13}, 5-\mu \mathrm{m}$-thick copper foils, ${ }^{14,15} 1-\mu \mathrm{m}$-thick copper thermistor foils, ${ }^{16}$ and 5 - $\mu$ m-thick nickel gauges ${ }^{17}$ were all studied. Based on conventional models of heat conduction, it is impossible to get microsecond time scale thermal equilibrium between the foil and the host material with these gauge thicknesses. Bloomquist and Sheffield ${ }^{11}$ proposed that the thermal conduction of polymethylmethacrylate (PMMA) in intense dynamic loading may be orders of magnitude faster than in normal loading (when the foil probe is perpendicular to the plane of the shock), but this proposal has neither been validated nor eliminated. Certainly this is a very interesting problem.

Recently, a foil thermopile (200 $\AA$ thickness) was successfully designed and used to measure the temperature rise in shock-compressed PMMA. ${ }^{18}$ This article describes follow up work in which $600-\AA \AA$-thick chromel-alumel foil thermocouples were used to measure temperature near a shaped charge generated cavity in medium carbon steel and glass fiber reinforced epoxy composite targets. Details relating to. the fabrication and use of these gauges are described.

\section{THEORETICAL ANALYSIS}

Several problems must be solved to measure temperature in shock-loaded solids with a foil thermocouple. First, the thermal inertia of the foil must be small enough that the real temperature of the host material can be measured on a microsecond time scale. Second, the effect of pressure on the thermopower, $S$, must be quantitatively determined because thermopower is a function of both temperature, $T$, and pressure, $P$. Hydrostatic compression experiments by Bridgman $^{19}$ and Bundy ${ }^{20}$ indicate that $\mathbf{S}(\mathbf{T}, \mathbf{P})$ can be written in the form

$$
\mathbf{S}(\mathbf{T}, \mathbf{P})=\mathbf{S}(\mathbf{T}, \mathbf{0})+\eta \mathbf{P},
$$

where $\eta$ is the coefficient of pressure. If the thermocouple material is carefully selected, the effect of pressure on thermopower can be kept below several percent.

If the experimental factors, such as dimensions of both target and projectile, impact velocity, etc., were all determined, shock pressure and its duration in the target could then be determined. In the case of the shaped charge jet penetration experiments described in this article, the conditions were not one dimensional so the profile and duration of the shock are unknown; loading duration was assumed to be in the range of $1-10 \mu \mathrm{s}$.

It was assumed that the host material and the foil thermocouple material were uniform and isotropic and that their thermal properties do not change as the temperature changes. The heat flux through the edges of the foil junction was assumed to be negligible in comparison to the heat flux through the flat surfaces. Both chromel and alumel are alloys 
TABLE I. Results of calculations.

\begin{tabular}{|c|c|c|c|c|c|}
\hline \multirow{2}{*}{$\begin{array}{l}\text { Foil } \\
\text { thickness }\end{array}$} & \multirow[b]{2}{*}{ Time } & \multicolumn{4}{|c|}{$T(x, t) / T_{0}(\%)$} \\
\hline & & $P M M A-N i^{a}$ & Composite $^{b}-\mathrm{Ni}$ & $2024 \mathrm{Al}-\mathrm{Ni}$ & Steel-Ni \\
\hline \multirow[t]{2}{*}{$a=300 \AA$} & $1 \mu s$ & 89.9 & $95.8^{-i}$ & 99.1 & 99.5 \\
\hline & $4 \mu \mathrm{s}$ & 95.7 & 98.5 & 99.7 & 99.8 \\
\hline \multirow[t]{2}{*}{$a=2.5 \mu \mathrm{m}$} & $1 \mu \mathbf{s}$ & 6.7 & 15.1 & 45.5 & 60.9 \\
\hline & $4 \mu \mathrm{s}$ & 13.0 & 27.4 & 65.9 & 78.9 \\
\hline
\end{tabular}

${ }^{a}$ Chromel and alumel are represented by $\mathrm{Ni}$.

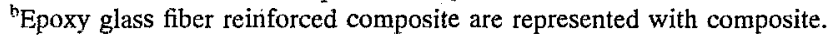

of nickel so the difference in their thermal properties was assumed to be negligible (in comparison with the properties of the host material) making it unnecessary to treal the foil as a two-part system. With these assumptions the problem could be modeled as a one-dimensional heat conduction problem. ${ }^{21,22}$ The characteristic time, $t_{c}$, in which a temperature change occurs is $\left(C_{v} \rho L^{2}\right) / \lambda$, where $C_{v}, \rho$, and $\lambda$ are specific heat, density, and heat conduction coefficients, respectively, and $L$ is the characteristic dimension. Using medium carbon steel as an example, the characteristic time for the foil thermocouple to be affected by material $2 \mathrm{~mm}$ away is $0.31 \mathrm{~s}$. This is much longer than the microseconds loading time, indicating that only the material very near the gauge affects the gauge measurement.

We were interested in determining how thin the foil thermocouple must be so that the temperature of the host material could be measured in a few microseconds long experiment. A one-dimensional heat conduction model with infinite boundaries was used. Initial conditions for the problem were that the temperature of the host material-foil thermocouple system were assumed to jump instantaneously from ambient values to their shocked value after the shock wave passed through them. At time zero the host has a temperature $T_{1}$ and the foil a temperature $T_{2}$. Since only the temperature rise, $T(x, t)$, in the foil is of interest, the temperature of the foil just after shock passage will be chosen as the zero of temperature axis and the temperature difference between the foil and the host material at time zero will be represented by $T_{0}=T_{1}-T_{2}$. [Notice that the real temperature of the foil is $T_{0}+T(x, t) .1$ The one-dimensional temperature diffusion equation with initial conditions $\left[T(x, 0)=T_{0},|x|>a ; T(x, 0)\right.$ $=0,-a<x<a]$ was used in our calculations, where " $a$ " was half the thickness of the thermocouple measuring junction. Since the foil was put on by vapor deposition, intimate contact between the foil and the host material is achieved. Therefore, we assume that the temperature and the heat flux are continuous at the interfaces $x=+a$ between the host and the foil.

The problem solution was obtained using Green's function and the "image" method. This process is similar to that used by Grover and Urtiew ${ }^{23}$ and Bloomquist and Sheffield. ${ }^{11}$ A solution with an infinite sum of error functions was obtained and an asymptotic series, was adopted to calculate the error function. The measured temperature of the foil thermocouple with respect to the host material can be expressed as a relative temperature rise, $T(x, t) / T_{0}$, going from 0 to 1 or as a percentage going from $0 \%$ to $100 \%$. (Although

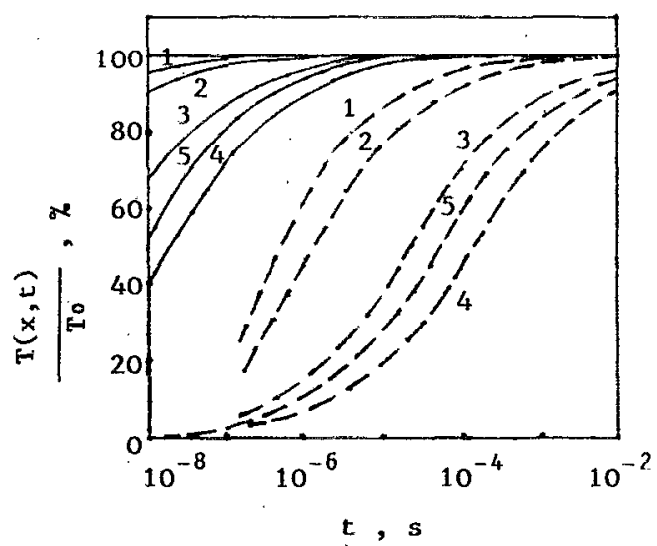

FIG. 1. Results from several heat conduction calculations in which the diffusion equation was solved to get the temperature vs time profile. The figure ordinate is the ratio of the temperature rise of the foil, $\mathbf{T}(x, t)$, divided by the difference in temperature between the foil and the host, $\mathbf{T}_{0}$, expressed as a percentage. The host materials and foil materials are noted by the numbers near each line: 1 for steel $\mathrm{Ni} ; 2$ for $2024 \mathrm{AI}-\mathrm{Ni} ; 3$ for epoxy composite-Ni; 4 for PMMA-Ni; and 5 for PMMA-Ag. " $a$ " is half the foil thickness of the measuring junction. Broken lines are for $a=2.5 \mu \mathrm{m}$ and solid lines are for $a=300 \AA$.

the ratio of measured foil temperature to the real host temperature would be $\left[T_{2}+T(x, t)\right] / T_{1}$, the ratio used is more suitable to indicate the change in foil temperature as a function of time, due to heat conduction.) If $T(x, t) / T_{0}=0$, the measured temperature is that of the shock-compressed foil. If $T(x, t) / T_{0}=1$, the measured temperature is that of the shock compressed host material.

The results of the calculations for several materials and thicknesses are given in Table I and Fig. 1. Material parameters used in the calculations are given in Table II. As can be seen, it is impossible for the foil and the host material to approach thermal equilibrium in a few microseconds when the foil is $5 \mu \mathrm{m}$ thick. For example, from Fig. 1 the calculated relative temperature rise percentage for a chromelalumel foil thermocouple $(a=2.5 \mu \mathrm{m})$ in $1 \mu \mathrm{s}$ is only $15 \%$ for a glass fiber reinforced epoxy composite host and $61 \%$ for a steel host material. However, this increases to $96 \%$ for the composite and $99.5 \%$ for the steel when $a=300 \AA$. This demonstrates the necessity of having an extremely thin foil thermocouple in our experiments.

Our thermocouple has a thickness $(2 a)$ of about $600 \AA$, i.e., the thickness of one foil material is $300 \AA$ and the junction is made up of two foil materials, one.on top of the other. The calculations indicate that the thermocouple temperature

TABLE II. Parameters of materials.

\begin{tabular}{lllll}
\hline Materials & \multicolumn{1}{c}{$\begin{array}{c}\rho^{*} \\
\mathrm{~g} / \mathrm{cm}^{3}\end{array}$} & $\begin{array}{c}C_{v} \\
\mathrm{Cal} / \mathrm{g} \mathrm{K}\end{array}$ & $\begin{array}{c}\lambda \\
\mathrm{Cal} / \mathrm{cm} \mathrm{s} \mathrm{K}\end{array}$ & $\begin{array}{c}D=\lambda / \rho C_{v} \\
\mathrm{~cm}^{2} / \mathrm{s}\end{array}$ \\
\hline Composite $^{\mathrm{b}}$ & 1.8 & 0.25 & 0.003 & 0.0067 \\
PMMA & 1.181 & 0.3511 & 0.00054 & 0.0013 \\
2024AJ & 2.785 & 0.2 & 0.049 & 0.088 \\
Steel & 7.9 & 0.105 & 0.108 & 0.13 \\
$\mathrm{Ni}^{\mathrm{a}}$ & 8.75 & 0.105 & 0.15 & 0.163 \\
\hline
\end{tabular}

${ }^{a}$ Chromel and alumel are represented by $\mathrm{Ni}$.

${ }^{b}$ Epoxy glass fiber reinforced composite are represented with composite. 
should be very near the temperature of the host material on the time scale of the experiment, thus providing a good measurement of the host material temperature.

\section{THE FOIL THERMOCOUPLE TECHNIQUE}

The selection of the proper thermocouple materials should take into account the temperature measurement range and the effect of pressure on thermopower. The expected thermoelectric emf can be obtained from the following cquation: ${ }^{9}$

$$
\begin{aligned}
\Phi_{b-a}=\Phi_{b}-\Phi_{a}= & -\int_{r_{r}}^{T_{j}} \mathbf{S}_{a-b}\left(\mathbf{T}^{\prime}, 0\right) d \mathbf{T}^{\prime}-\frac{1}{2} \mathbf{P}\left(\eta_{a} \eta_{b}\right) \\
& \times\left(\mathbf{T}_{j}-\mathbf{T}_{r}\right)
\end{aligned}
$$

where $\Phi$ and $T$ are electric potential and temperature, respectively, and $\mathbf{S}(\mathbf{T}, \mathbf{P})$ is the thermopower of the thermocouple. The subscripts $a$ and $b$ refer to the two materials comprising the thermocouple, i.e., chromel and alumel. $\mathbf{T}_{j}$ and $\mathbf{T}_{r}$ are the temperatures at the measuring and reference junctions, respectively. The first term in Eq. (2) expresses the electric potential associated with the temperature difference at normal pressure. It can be related to the temperature at the point of interest with the aid of a standard thermocouple table. The second term represents the contribution of the pressure to the thermoelectric emf. The pressure coefficient for several different thermocouple materials has been reported by Bundy. ${ }^{20}$ The pressure coefficient for a chromel-alumel thermocouple is

$$
\eta_{\mathrm{Cr}}-\eta_{\mathrm{Al}}=0.12 \mu \mathrm{v} \mathrm{GPa}{ }^{-1} \mathrm{~K}^{-1}
$$

and is less than the other materials. This pressure correction corresponds to about a $3 \%$ decrease in the expected emf at $10 \mathrm{GPa}$. Because of this and also the fact that temperatures up to $1573 \mathrm{~K}$ can be measured with this type of thermocouple, chromel and alumel were chosen as the thermocouple materials to be used in these experiments.

In order to make a foil thermocouple with a thickness of $600 \AA$, a vacuum deposition technique was used. Each of the thermoelectrode materials making up the thermocouple was deposited on the target separately. The reference junction was put on the lateral surface. The configuration is shown in Fig. 2. The material making up the foil thermocouple was deposited by heating a tungsten wire on which the chromel or alumel was wound, in order to evaporate it. Tungsten wire was selected as the heater because of its high melting point and its high strength at elevated temperatures. Each tungsten heater was used only once because there was a possibility of it alloying with the material being deposited which would reduce its melting point.

Each thermocouple involved four different depositions, two for each material. One deposition made one side of the thermocouple and then the sample was relocated and a second deposition made on the lateral surface. The same process was used to deposit the other side of the thermocouple. The edge of the plate the thermocouple was being deposited on, between the side and the face, was rounded prior to the deposition process to assure a good connection between the two depositions.

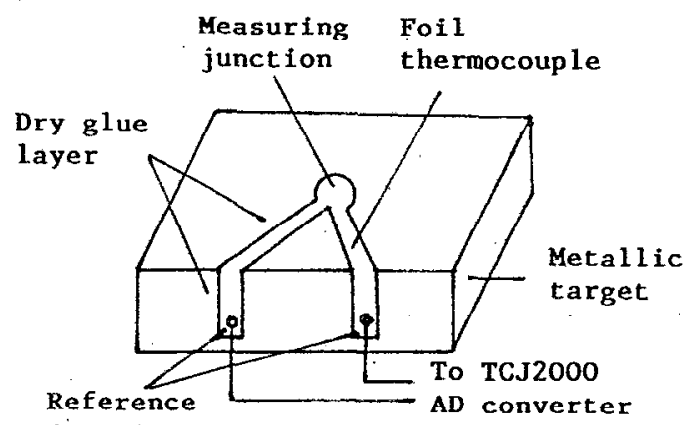

junction

FIG. 2. Sketch of the measuring temperature plate with the thermocouple in place. The diameter of the measuring junction is $3 \mathrm{~mm}$. The biggest width of the thermoconple leads is $15 \mathrm{~mm}$. The radius of the plate edge at the leads connection is $3 \mathrm{~mm}$.

Deposition thickness was controlled by controlling the current and voltage of the tungsten heater and the time it was heated. The relationship between these parameters and the thickness was determined in advance. This was done by means of a carving line mark and multiple beam interferometry by the Chinese Metrology Institute. It was found that the thickness was directly related to the resistance when the width was constant, so the resistance was used as a measure of the thickness on most of the deposition.

There were concerns about selective evaporation of the components of chromel and alumel. However, an analysis of the starting wire material and a deposited layer demonstrated the composition was the same. The deposited sample was made by doing a number of depositions because a relatively thick layer was required to do the analysis. The analysis (including spectrum analysis and microanalysis) was done by the Beijing Central and Steel Research Institute. A calibration of thermocouple emf versus temperature for a deposited thermocouple (to be discussed later) also confirmed this.

For the metal target, insulation between the thermocouple leads must be ensured in order to measure an emf. This was accomplished by coating the polished and cleaned surface of the metal with a 1- $\mu$ m-thick layer of CS212 epoxy (supplied by the Chunsu Co.), including the lateral surface on which the reference junction was deposited (see Fig. 2). The place where the junction of the thermocouple was to be deposited was not coated with glue so that the measuring junction would be in direct contact with the metal. One lead (thermoelectrode) of the thermocouple was deposited on the epoxy with the measuring junction being deposited on the metal. Following this the other lead (other thermoelectrode) of the thermocouple was deposited as shown in Fig. 2. This assembly (except for the measuring junction) was coated with the dilute fresh CS212 epoxy with a thickness of $1 \mu \mathrm{m}$. By this method the measuring junction was in direct contact with the metal target (on both sides) but the thermocouple leads (including on the lateral surface) were insulated.

The design was such that the measuring junction was in the shock-compressed region of the target and the reference junction, as shown in Fig. 2, was on the lateral surface of the target (which was always at ambient pressure and ambient temperature and was convenient for connecting the output 
TABLE III. Results of calibrating foil thermocouple.

\begin{tabular}{|c|c|c|c|c|c|}
\hline \multirow[b]{2}{*}{ Thermocouple (mv) } & \multicolumn{5}{|c|}{ Temperature (K) } \\
\hline & 473 & 673 & 873 & 1073 & 1273 \\
\hline Standard chromel-alumel & 8.13 & 16.40 & 24.90 & 33.29 & 41.27 \\
\hline Foil chromel-alumel & 8.11 & 16.34 & 24.80 & 33.18 & 41.14 \\
\hline
\end{tabular}

leads). A similar thermocouple metal plate assembly was made for measuring the electromagnetic disturbance during the experiment.

The composite target was an insulator so the foil thermocouple was directly deposited on it. The method of vacuum deposition of the foil thermocouple was the same as for the metal target.

Several experiments were done to confirm that this foil thermocouple design could reliably measure temperature. First, it was shown that the chemical composition of the chromel and alumel were the same before and after vacuum deposition. Second, it was determined that the emf was zero in the circuit if both poles of the thermocouple were made of the same material (chromel, alumel, or aluminum) and the thermocouple was subjected to one-dimensional shock loading. Third, in order to calibrate the thermal emf versus temperature for the foil thermocouple, chromel and alumel were vacuum deposited separately on a long sheet of alumina. The measuring junction was kept in a high temperature furnace with pure nitrogen protection. The reference junction was at ambient temperature. The temperature of measuring junction and reference junction were always monitored by Le Chatelier thermocouples. The results of the calibration basically agree with the standard thermocouple (see Table III). These confirmation experiments were reported in detail in Ref. 18.

\section{EXPERIMENTAL SETUP}

A sketch of the medium carbon steel experiment is shown in Fig. 3. The size of the medium carbon steel plates used for the target pieces was $120 \mathrm{~mm}$ by $120 \mathrm{~mm}$ by $20 \mathrm{~mm}$ thick. The experiment consists of several plates stacked one on top of the other as shown in Fig. 3. The two-plate assembly (called the measuring temperature plates) where the temperature was measured was put at a particular position in the experimental assembly. This position was where the speed of the jet element penetrating the measuring temperature plate was $5.4 \pm 0.1 \mathrm{~km} / \mathrm{s}$ (sce the shaped charge discussion below). Several nearly identical experiments were done with the only difference being the thermocouple distance from the shaped charge jet axis.

In order to determine the magnitude of any electrical disturbance and be able to subtract it out of the measuring thermocouples emf, a two-plate assembly (called the measuring disturbance plates) was made with a thermocouple inside it. It was put on the side of the experiment in a position where it was not affected by the shock loading but it was subjected to any electromagnetic disturbances that might be present. The positioning of this set of plates is shown in Fig. 3.

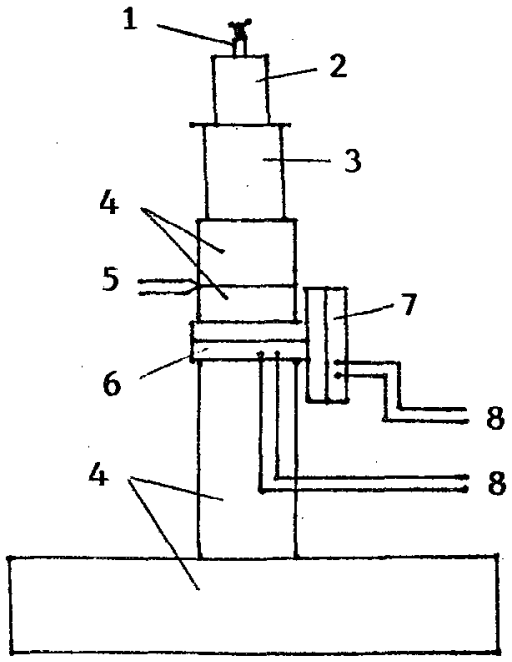

FIG. 3. Experimental configuration for the shaped charge jet penetration experiment showing the measuring temperature target plate in place. The thermocouple was sandwiched between the two medium carbon steel plates. Two measuring disturbance plates with a thermocouple sandwiched between them are shown mounted on the periphery of the measuring temperature plates. A description of the various parts is: 1 -detonator; 2-shaped charge (diameter $82 \mathrm{~mm}$ ); 3 -standoff spacer (height $164 \mathrm{~mm}$ and diameter 90 $\mathrm{mm})$; 4-bottom plate and base; 5-trigger generator for $\mathrm{AD}$ converter; 6-measuring temperature plates; 7-measuring disturbance plates (the plate size was $120 \times 120 \times 20 \mathrm{~mm}$ ); and 8 -leads to TCJ $-2000 \mathrm{AD}$ converter.

The composite target experimental setup was similar to the medium carbon steel experiments. All the plates were $120 \mathrm{~mm}$ by $120 \mathrm{~mm}$ by $20 \mathrm{~mm}$ thick, including the composite plates. The target buildup was the same with the medium carbon steel plates in front of and behind the measuring temperature plates. Only the two-plate assemblies for the measuring temperature plates and the measuring disturbance plates were made from the composite material. By setting up the experiment in this way, the speed of the jet element penetrating the composite target was still $5.4 \mathrm{~km} / \mathrm{s}$. Several nearly identical experiments were completed with the only difference being the thermocouple distance from the shaped charge axis.

Small metallic pieces hooked to the lead wires were used to make the electrical connection between the lead wires and the thermocouple leads because they were so delicate. The connection parts between the lead wires and the thermocouple leads was the reference junction of the thermocouple. Nylon screws were used to hold the metallic pieces solidly on the leads.

The voltages generated by the thermocouples in the measuring plates and the disturbance plates were measured with two TCJ-2000 transient converters (sampling time was 0.1 $\mu$ s/word, memory circuit 2048 words, response speed 60 ns, DA conversion speed $50 \mathrm{~ns}$ ) and a Tektronix 7834 oscilloscope or type TR-4653 HF oscilloscope. The oscilloscopes were only used to display the records. Resolution of the measured voltages on the transient converters was $512 \pm 2$ levels for the full scale voltage resulting in a temperature resolution of about $2.5 \mathrm{~K}$. Sampling time was $0.1 \mu$ s per point. Voltage sensitivities of 50 and $100 \mathrm{mV}$ full scale were usually used, 
depending on the distance the thermocouple was from the shaped charge axis.

All the possible electrical and magnetic disturbances were analyzed in advance and eliminated as much as possible. All the wires (signal, trigger, and ignition) were shielded cables. The two-plate assemblies (measuring temperature plates and measuring disturbance plates) were wrapped up in $50-\mu$ m-thick aluminum foil which was grounded. Even so, there was an electromagnetic disturbance generated by the explosive charge. The measured emf coming from the thermocouple in the measuring temperature plates was the sum of the thermocouple emf and the disturbance emf, but since the disturbance was measured by the thermocouple circuit in the disturbance measuring plate, it could be subtracted out to yield the true thermocouple emf.

In order to obtain a high speed jet, a standard shaped charge was designed for the experiments. The liner of the shaped charge was made of copper. The jet speed along its axis continuously varied from $7 \mathrm{~km} / \mathrm{s}$ at the head to $1.5 \mathrm{~km} / \mathrm{s}$ at the tail. A number of experiments (over 20) were done to determine the penetration depth in the medium carbon steel target. The penetration depth had a standard deviation of only $1.6 \%$ of the total penetration depth. In order to obtain consistent experimental results the experimental conditions were carefully controlled. The shock pressure around the penetrating point on the measuring temperature plate depends on the speed of the jet element interacting with the plates. In our experiments the speed of the jet element penetrating the measuring temperature plate was controlled at $5.4 \pm 0.1 \mathrm{~km} / \mathrm{s}$. The jet elements over $5.4 \mathrm{~km} / \mathrm{s}$ were exhausted by the steel target plates above the measuring temperature plates. The speed of the jet element penetrating the medium carbon steel plates was measured by means of a high speed sweep camera, four sets synchro-pulse $x$ radiography (interval $5 \mu \mathrm{s}$ ), and electrical measurement. The jet speed was measured in setup experiments and not in the actual thermocouple experiments.

\section{RESULTS AND DISCUSSION}

Voltage versus time wave forms obtained from the medium carbon steel experiments are shown in Fig. 4. These

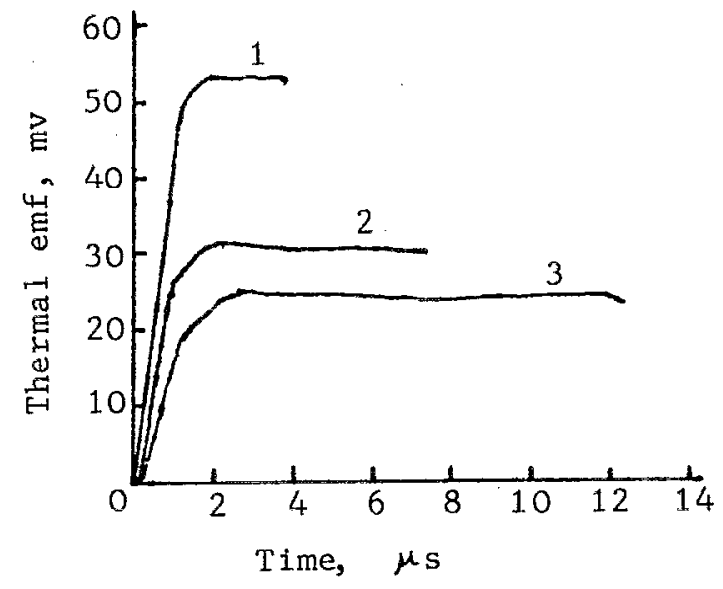

FIG. 4. Voltage signal vs time at different distance from cavity center, $1-5.4$ $\mathrm{mm} ; 2-9.8 \mathrm{~mm}, 3-11.1 \mathrm{~mm}$. These wave forms were obtained from the medium carbon steel experiments.
TABLE IV. Results of measured temperature in medium carbon steel targets.

\begin{tabular}{cccc}
\hline \hline No. & $\begin{array}{c}\text { Distance from } \\
\text { cavity center } \\
(\mathrm{mm})\end{array}$ & $\begin{array}{c}\text { Measure } \\
\text { emf } \\
(\mathrm{mv})\end{array}$ & $\begin{array}{c}\text { Temperature } \\
(\mathrm{K})\end{array}$ \\
\hline 1 & 5.4 & 51.7 & 1554 \\
2 & 9.8 & 30.3 & 1001 \\
3 & 11.1 & 24.9 & 873 \\
\hline \hline
\end{tabular}

wave forms were obtained in three different experiments with the measuring thermocouples different distances from the shaped charge axis. The nearer the thermocouple was to the axis, the higher the thermal emf. Because of the increasingly harsh environment the thermocouple was subjected to as the position gets nearer to the shaped charge axis, the measurement time decreases. This can be attributed to the higher shock pressures and increased plastic deformation as the axis of the shaped charge is approached. Large plastic deformation causes the thin foils to break. A post-experiment examination of the recovered temperature measuring plates indicated this was the case.

The temperatures resulting from the emfs on the flat part of the wave forms are listed in Table IV and plotted in Fig. 5. They were determined from standard chromel-alumel thermocouple tables. It was not possible to determine the pres-

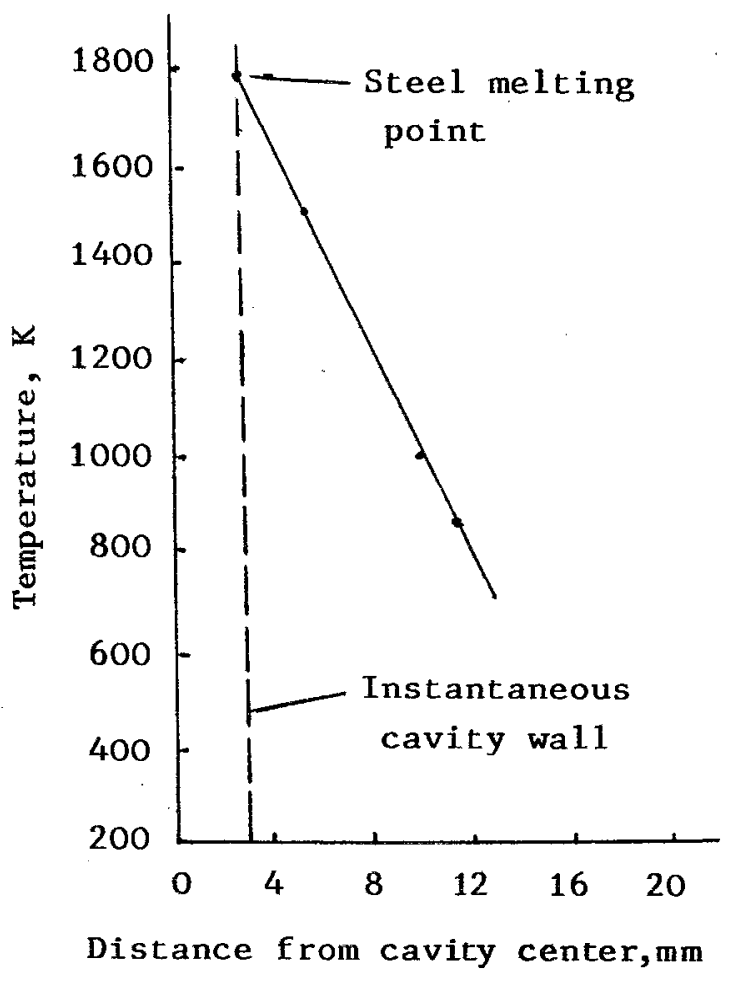

FIG. 5. Temperatures measured at different distances from the shaped charge jet axis in the medium carbon steel plates. Black points denote the experimental data. Instantaneous cavity wall dimension means cavity diameter at the time of early penetration. This was measured by four synchropulse $\mathrm{x}$-radiography cameras. The jet diameter was $2.8 \mathrm{~mm}$; instantaneous cavity diameter was $7 \mathrm{~mm}$; final cavity diameter was $12 \mathrm{~mm}$. On the final cavity wall there were fish-scale melting patterns which were the result of inertial motion of the melting layer at late experimental times. 
TABLE V. Time of thermal degradation under different temperature (s).

\begin{tabular}{|c|c|c|c|c|c|}
\hline \multirow{2}{*}{$\begin{array}{c}\text { Temperature } \\
\text { (K) }\end{array}$} & \multicolumn{5}{|c|}{ Reaction percent $x$} \\
\hline & $10 \%$ & $30 \%$ & $50 \%$ & $70 \%$ & $90 \%$ \\
\hline 723 & $1.19 \times 10^{-6}$ & $4 \times 10^{-6}$ & $7.8 \times 10^{-6}$ & $1.35 \times 10^{-5}$ & $2.6 \times 10^{-5}$ \\
\hline 823 & $9.7 \times 10^{-9}$ & $3.3 \times 10^{-8}$ & $6.4 \times 10^{-8}$ & $1.5 \times 10^{-7}$ & $2.1 \times 10^{-7}$ \\
\hline 973 & $5.6 \times 10^{-10}$ & $1.9 \times 10^{-9}$ & $3.7 \times 10^{-9}$ & $8.5 \times 10^{-9}$ & $1.2 \times 10^{-8}$ \\
\hline
\end{tabular}

sure at the measuring point so it was not reasonable to make a pressure correction to the emf. However, this is known to be less than a few percent so the effect was neglected. The ambient temperature at the time of the experiment was taken as the temperature of the reference junction.

A post-experiment examination of the steel plates indicated evidence of steel melting at the edge of the cavity. For this reason a dashed line was extended from the measured data to the melting temperature at the cavity wall in Fig. 5. Using this point and the three data points, a straight line fits through them quite well. As was expected the temperature during shaped charge penetration drops off rapidly as the measurement position moves away from the cavity wall.

An indirect method was used to roughly calibrate the measured thermocouple temperature to provide a basis for believing the measurements. It is well known that thermal degradation of epoxy occurs at a certain temperature that can be determined in a thermal degradation experiment. If the temperature measured by the thermocouple at the position where the thermal degradation started to occur is close to that determined in the thermal degradation experiment, a rough calibration of the thermocouple measurement results. This is the reason the glass fiber reinforced epoxy composite target experiments were carried out. The composite plates were used in place of the medium carbon steel measuring temperature plates. After the shaped charge jet penetrated the composite, a post-experiment examination of the composite plates was used to determine the region where the epoxy was beginning to be degraded. ${ }^{24}$

Thermal degradation experiments and calculations were completed on the epoxy composite material to determine the temperature/time relationship for degradation to occur. These tests indicated that the temperature of the incipient degradation was $723 \mathrm{~K}$. Static pyrolytic tests were run on the composite and they showed that degradation occurs in the temperature range from 723 to $1033 \mathrm{~K}$, with the amount of gaseous products increasing as the temperature increases. Calculations were done to obtain the theoretical relationship between degradation and time as given in Table $V$. The higher the temperature, the greater the degradation and the faster the degradation occurs. At $823 \mathrm{~K} 90 \%$ degradation occurs in $2.1 \times 10_{-}^{-7} \mathrm{~s}$.

Examination of the measuring temperature composite plates after the experiment indicated that epoxy degradation occurred between the cavity wall and $5 \mathrm{~mm}$ in. On the cavity wall and in the cracks around the cavity wall, a mixture of carbon and tar occurs. The formation of this carbon/tar mixture starts $5 \mathrm{~mm}$ from the cavity wall and the closer to the wall the position being looked at is, the more of the mixture

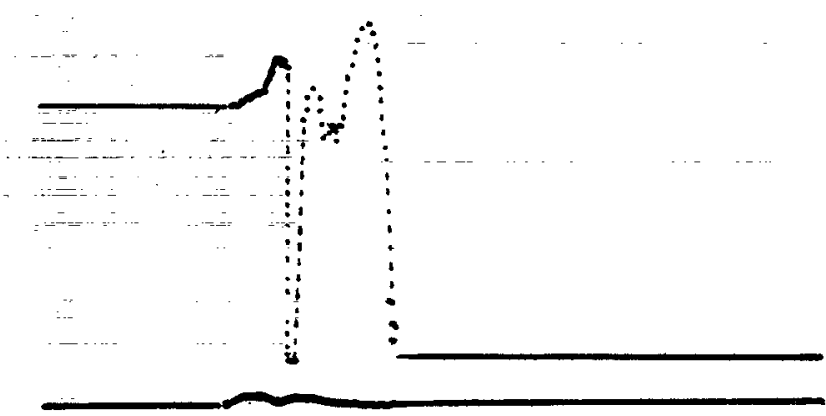

FIG. 6. Photograph of the oscilloscope traces showing the voltage signal from the measuring disturbance thermocouple in the lower traces $(2.8 \mathrm{mV})$ and the measuring temperature thermocouple in the upper trace $(20.3 \mathrm{mV})$ in the composite target. The measured thermal emf use $17.5 \mathrm{mV}$ with the peak sustained for $2.2 \mu \mathrm{s}$. Because the composite target was a brittle material undergoing thermal degradation, the thermocouple leads were rapidly broken by the dynamic deformation process.

is evident. This indicates that the temperature $5 \mathrm{~mm}$ in from the cavity should have been about $723 \mathrm{~K}$.

A set of TR-4653 HF oscilloscope voltage wave forms obtained from one of the composite experiments is shown in Fig. 6 . The upper wave form was obtained from the measuring temperature plate thermocouple and the lower wave form from the disturbance plate thermocouple. At the beginning the emf rises slowly on both the upper and lower wave forms. Then the lower wave form (disturbance) decreased to zero because the emf from the disturbances went to zero. The

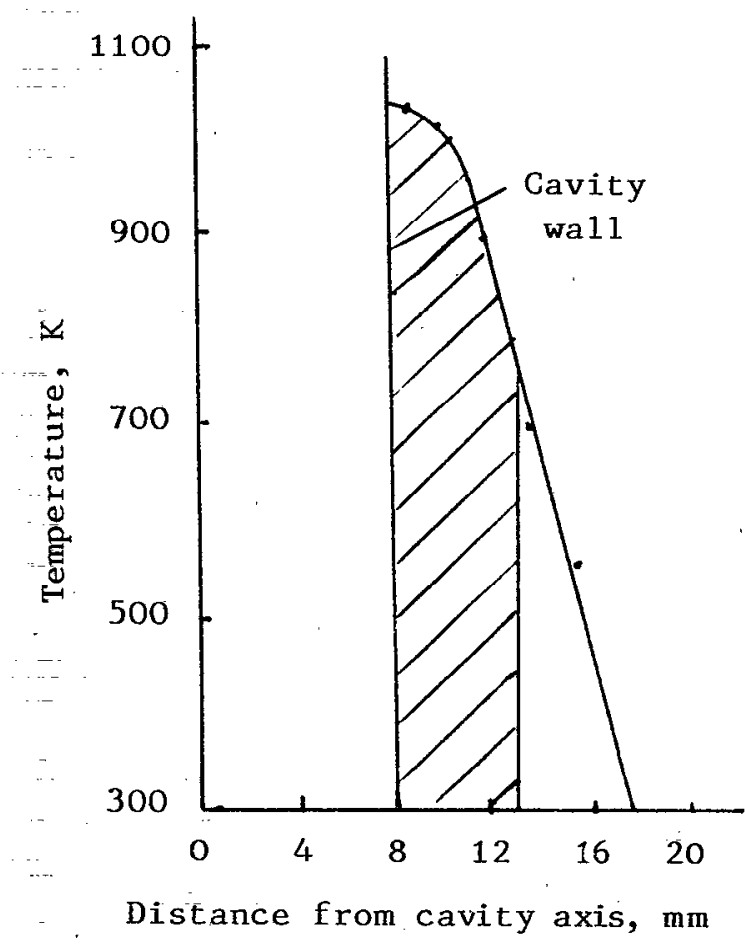

FIG. -7. Temperatures measured at different distances from the shaped charge jet cavity axis in the fiber glass reinforced epoxy composite. The thermal degradation area, as determined from a post-experiment examination of the plates, is shown as the crosshatched area. Black points denote the experimental data. The radius of the cavity in the composite target was 8 $\mathrm{mm}$. 
TABLE VI. Results of measured temperature in epoxy glass fiber reinforced composite.

\begin{tabular}{lccc}
\hline \hline No. & $\begin{array}{c}\text { Distance from } \\
\text { cavity wall } \\
(\mathrm{mm})\end{array}$ & $\begin{array}{c}\text { Measure } \\
\text { emf } \\
(\mathrm{mv})\end{array}$ & $\begin{array}{c}\text { Temperature } \\
(\mathrm{K})\end{array}$ \\
\hline 1 & 0.8 & 31.6 & 1032 \\
2 & 2.0 & 30.6 & 1008 \\
3 & 3.4 & 25.9 & 897 \\
4 & 5.2 & 17.5 & 699 \\
5 & 7.3 & 11.6 & 557 \\
\hline \hline
\end{tabular}

upper wave form increased up to a peak, leveled off for several microseconds, and then decreased to zero because the foil was broken due to plastic deformation (as determined by post-experiment examination of the composite plates). To get the true emf wave form, the lower trace was subtracted from the upper trace.

Measured temperatures versus position from the cavity wall are given in Table VI and plotted in Fig. 7 for the glass fiber epoxy composite experiments. In Fig. 7 the region where the carbon/tar mixture was detected in the postexperiment examinations has been shaded, i.e., the region from 8 to $13 \mathrm{~mm}$ from the cavity axis. When a line is drawn through the data, it appears that the start of composite degradation intersects the line at about $750 \mathrm{~K}$. This is a very nice correlation with the static tests which indicate degradation starts at about $723 \mathrm{~K}$. These experiments represent independent confirmation that the temperatures being measured are reasonable.

It has been theoretically and experimentally shown that measuring temperature in shock-loaded solids on a microsecond time scale is feasible if a $600-\AA$-thick chromel-alumel thermocouple is used. The thermal equilibration time is sufficiently short to allow a measurement to be made if the experiments are carefully prepared. An experimental method has been developed and used to measure the temperature distribution in shaped charge jet loaded plates of medium carbon steel and glass fiber reinforced epoxy composite. The measurements for both materials are in the temperature range expected; $870-1550 \mathrm{~K}$ for the steel and $560-1030 \mathrm{~K}$ for the composite. The measurements were indirectly calibrated by using post-experiment examination of the steel and composite plates, along with other thermal degradation experiments in the latter case.

\section{ACKNOWLEDGMENTS}

The authors wish to thank Chemin Cheng, Qingmin Tan, and Jiachun Lee for helpful discussions, and Xiaohong Xu for much help. This work was sponsored by National Advance Materials Committee of China, the China National Science Foundation Committee, and the Laboratory for Mechanics of Materials Processing, Institute of Mechanics, Chinese Academy of Sciences.

${ }^{1}$ G. E. Duvall, in Shock Waves in Condensed Matter, edited by Y. M. Gupta (Plenum, New York, 1986), pp. 1-12.

${ }^{2}$ R. A. Graham, J. Phys. Chem. 83, 3084 (1979).

${ }^{3}$ G. A. Lyzenga and T. J. Ahrens, Bull. Am. Phys. Soc. 24, 714 (1979).

${ }^{4}$ P. A. Urtiew and R. Grover, J. Appl. Phys. 48, 1122 (1979).

${ }^{5}$ W. G. Von Holle and E. L. Lee, Proceedings of the International Symposium on the Behavior of Dense Media Under High Dynamic Pressures (Commissariats à l'Encrgia Atomique, Paris, 1978), pp. 425-438.

${ }^{6}$ C. S. Coffey and S. J. Jacobs, J. Appl. Phys. 52, 6991 (1981).

${ }^{7}$ S. W. Yuan and J. P. Billingsley, Appl. Sci. Res. 24, 32 (1971).

${ }^{8}$ A. A. Gelibas, V. A. Simonov, and I. D. Fakzalunk, Proceedings of the Fifth International Conference on High Energy Rate Fabrication, Denver, CO, 1975, edited by I. V. Yakovlev and V. F. Nesterenko, pp. 4.1,1.

${ }^{9}$ D. D. Bloomquist, G. E. Duvall, and J. J. Dick, J. Appl. Phys. 50, 4838 (1979).

${ }^{10}$ S. A. Sheffield and D. D. Rloomq̨uist, Bull. Am. Phys. Snc. 25, 567 (1980).

${ }^{11}$ D. D. Bloomquist and S. A. Sheffield, J. Appl. Phys. 51, 5260 (1980).

${ }^{12}$ D. D. Bloomquist and S. A. Sheffield, J. Appl. Phys. 53, 5966 (1982).

${ }^{13}$ Z. Rosenberg and Y. Partom, J. Appl. Phys. 55, 3999 (1984).

${ }^{14}$ D. D. Bloomquist and S. A. Sheffield, Appl. Phys. Lett. 38, 185 (1981).

${ }^{15}$ D. D. Bloomquist and S. A. Sheffield, Bull. Am. Phys. Soc. 26, 257 (1981).

${ }^{16}$ Z. Rosenberg and Y. Partom, J. Appl. Phys. 56, 1921 (1984).

${ }^{17}$ Z. Rosenberg and Y. Partom, J. Appl. Phys. 52, 6133 (1982).

${ }^{18}$ Juxian Gao, Rongshang Bai, and Chemin Cheng, J. Appl. Phys. 67, 2272 (1990).

${ }^{19}$ P. W. Bridgman, Proc. Am. Acad. Arts. Sci. 53, 269 (1918).

${ }^{20}$ F. P. Bundy, J. Appl. Phys. 32, 483 (1961).

${ }^{21}$ A. H. Techonof and A. A. Sarmarlskee, $A$ Course in Equation of Mathematical Physics (National Technology-Theoretical Press, Moscow, 1953) (USSR).

${ }^{22}$ H. S. Carlslaw and J. C. Jaeger, Conduction of Heat in Solids, 2nd ed. (Oxford University Press, London, 1959).

${ }^{23}$ R. Grover and P. A. Urtiew, J. Appl. Phys. 45, 146 (1974).

${ }^{24}$ Cheng Chemin, Tan Qingming, Gao Jusian, Ye Dongying, Wu Yuyan, Proceedings of the International Symposium on Intense Dynamic Loading and Its Effects, edited by Cheng Chemin and Ding Jing (Science Press, Beijing, 1986), pp. 347-351. 